\title{
Diagnosi di infezione acuta primaria da HIV in donatore di sangue
}

\section{Rosa Anna Leone', Pasquale Minchella', Salvatore Nisticò', Giuseppe Ivan Potente', Francesco Mellea ${ }^{2}$, Domenico Caruso', Mario Camerino', Barbara Gagliardi', Caterina Mustaro',Antonella Nicolazzo', Angela Luciano'}

' U.O. Microbiologia e Virologia, Azienda Sanitaria n. 6, 88046 Lamezia Terme (CZ)

2 U.O. Otorinolaringoiatria, Azienda Ospedaliera "Pugliese-Ciaccio", 88100 Catanzaro

Key words: test screening HIV, primary infection, p24

\section{Case report: early detection of symptomatic primary HIV infection}

\section{SUMMARY}

The early detection of HIV infection is of paramount either to achieve a better blood supply safety and to pose a prompt diagnosis so that to decrease the involuntary spreading of the disease. The fourth generation screening assays, which measure both the levels of the anti-HIV antibodies and the p-24 viral antigen, and mainly the development and widespread usage of the molecular biology tests, which identify the HIV-RNA in the early stage, have further reduced the diagnostic window period. We report a case about an acute primary HIV infection, occurred in a periodic donor, promptly diagnosed by the combined screening assays and the detection of HIV-RNA using molecular tests.

\section{INTRODUZIONE}

Negli ultimi dieci anni in Italia, così come negli altri Paesi europei, l'incidenza di nuovi casi di AIDS è diminuita, soprattutto grazie al miglioramento delle tecniche diagnostiche ed all'impiego di combinazioni di farmaci antiretrovirali in grado di dare una maggiore probabilità di risposta virologica ottimale.

Bisogna comunque sempre essere molto vigili e tenere alta l'attenzione sul possibile rischio di trasmissione di HIV attraverso trasfusione di sangue ed emoderivati nel periodo finestra.

Lo screening sierologico dell'infezione da HIV si basava, fino a qualche anno fa, sulla ricerca di anticorpi anti-HIV con tecnica EIA; esisteva tuttavia un periodo di circa 3-4 settimane (finestra sierologica), tra contagio e comparsa degli anticorpi, in cui però nessun segno di infezione poteva essere rilevato.

Successivamente sono stati sviluppati dei test di screening sierologico di quarta generazione che, combinando la rilevazione degli anticorpi antiHIV con quella dell'antigene virale p24, hanno portato ad una riduzione della finestra diagnostica. Secondo dati dell'Istituto Superiore di Sanità fra i nuovi casi di AIDS quasi una persona su due scopre di essere sieropositiva al momento della diagnosi di malattia conclamata o poco prima: per un periodo medio di circa dieci anni, quindi, gran parte dei sieropositivi resta inconsapevole del proprio stato di potenziale contagiosità, con gravi conseguenze sulla possibile diffusione dell'infezione.

La categoria maggiormente colpita negli ultimi anni è quella degli eterosessuali (40\%) seguita dai tossicodipendenti $(32 \%)$ e dagli omosessuali $(20 \%)$.

Il sospetto di infezione acuta da HIV dovrebbe portare ad effettuare indagini sia sierologiche (indirette) che virologiche (dirette): infatti la sierologia inizialmente è negativa, con sieroconversione successiva; la diagnosi nelle fasi precoci dell'infezione è, quindi, soprattutto diretta mediante rilevazione dell'antigene p24 con metodica EIA o meglio mediante determinazione della carica virale (HIV-RNA) con tecniche di biologia molecolare.

Il rischio residuo di trasmissione di HIV con donazioni di sangue effettuate nel periodo finestra, possibilità comunque reale, anche se poco frequente, oggi si è significativamente ridotto giacché è diventato obbligatorio sottoporre il sangue donato alla ricerca dell'HIV-RNA con metodica di biologia molecolare.

Descriviamo un caso di infezione acuta da HIV nella fase di sieroconversione, diagnosticato tempestivamente.

Non è frequente né facile diagnosticare un'infezione acuta da HIV in quanto il sospetto diagnostico è spesso fuorviato da manifestazioni cliniche 
varie, non specifiche e da una anamnesi che molte volte, per reticenza del paziente, non mette in evidenza fattori di rischio.

\section{Descrizione del caso clinico}

Un paziente di sesso maschile di 26 anni, donatore periodico, è stato ricoverato il 22 maggio 2003 presso l'U.O. di Malattie Infettive per astenia, febbre, mal di gola e disturbi gastrointestinali. Il paziente riferiva di avere già da alcuni giorni uno stato febbrile, con malessere generalizzato, tosse notturna non produttiva e diarrea. L'esame obiettivo metteva in evidenza una faringo-tonsillite essudativa, una linfoadenopatia laterocervicale ed una dermatite con esantema maculare soprattutto nella regione del tronco. Sono stati pertanto eseguiti prelievi ematici per emocromo, esami biochimici tra cui enzimi epatici, esami coagulativi, esami sierologici per Epatite B, Epatite C, HIV, lue, mononucleosi, varicella e tamponi faringeo, rettale e cutaneo. Gli esami di laboratorio dimostravano lieve linfopenia e trombocitopenia con enzimi epatici nella norma; gli esami sierologici per Epatite B, Epatite C, lue e mononucleosi erano negativi. Solo ad una indagine anamnestica più accurata il paziente rivelava di essere omosessuale, di aver cambiato partner da circa due mesi ed inoltre di aver effettuato una donazione il 6 maggio dello stesso anno. Quindi, per quanto riguarda il test sierologico per l'HIV, è stato effettuato dapprima il test di screening utilizzato all'epoca, cioè il test $\mathrm{HIV} 1 / 2 \mathrm{gO}$ Axsym, metodo Meia (Abbott) che è risultato "Non reattivo", con indice $\mathrm{S} / \mathrm{CO} 0.45$ (positivo > 1.0); successivamente sullo stesso prelievo, considerato il forte sospetto di sindrome simil-mononucleosica da infezione acuta da HIV, è stato effettuato il test sierologico HIV Vidas Duo, metodo ELFA (bioMérieux) per la ricerca dell'antigene p24, oltre che per gli anticorpi anti-HIV 1-2, non utilizzato di solito nella routine perché non completamente automatizzato. Tale test ha dato risultato positivo limite, con indice 0.26 (negativo $<0.25$; positivo limite 0.25 -
0.35; positivo $>0.35$ ). Visti i risultati, il giorno dopo (23 maggio 2003) veniva ripetuto il prelievo per il test sierologico per HIV e contemporaneamente veniva effettuata la ricerca dell'HIV-RNA su plasma. Il test HIV Duo di quarta generazione risultava positivo (indice 0.41 ), come pure il test HIV gO di terza generazione (indice 1.59); inoltre il test Cobas Amplicor HIV-RNA Monitor, metodo PCR quantitativa (Roche Diagnostics) dava un risultato positivo riguardo la viremia $(110000$ copie/ml) (tabella 1).

\section{DISCUSSIONE}

Nel caso clinico descritto il paziente si presentava febbrile, con una sindrome multisistemica ed una eruzione cutanea polimorfa, ma con una prima anamnesi senza fattori di rischio, avendo egli negato recenti viaggi, uso di droghe e rapporti sessuali a rischio. Solo il forte sospetto diagnostico di sindrome simil-mononucleosica da infezione acuta da HIV, scaturito da un'indagine anamnestica più accurata che ha rivelato fattori di rischio, ha permesso di mettere in evidenza la sieroconversione. L'uso di sistemi diagnostici sensibili e specifici, come i test sierologici combinati di quarta generazione che ormai sono in uso di routine al posto dei kit di terza generazione ed $\mathrm{i}$ test per la ricerca di HIV-RNA con tecniche di biologia molecolare, offrono oggi la possibilità di effettuare una diagnosi virologica di infezione acuta primaria. Nel caso descritto i componenti della donazione sono stati rapidamente rintracciati: i globuli rossi risultavano trasfusi ed il plasma ritirato dall'industria alla quale era stato consegnato per ottenere prodotti di derivazione. Fortunatamente il ricevente è risultato negativo sia per HIV-RNA che per anticorpi anti-HIV a distanza di 3, 6, 9 e 12 mesi dalla trasfusione; la ricerca di HIV-RNA sul plasma della donazione del 6 maggio ha dato anche esito negativo. In conclusione, la diagnosi precoce della sindrome acuta da HIV è di grande importanza per limitare la trasmissione inconsapevole dell'infezione.

Tabella I. Risultati test sierologici e ricerca di HIV-RNA (PCR)

\begin{tabular}{lllll}
\hline DATA & METODO & RISULTATO & VALORE & Cut-off \\
\hline $22 / 05 / 2003$ & MEIA (S/CO) & Non reattivo & $\mathbf{0 . 4 5}$ & Positivo $>$ I.0 \\
\hline $22 / 05 / 2003$ & ELFA & Positivo Limite & $\mathbf{0 . 2 6}$ & $\frac{\text { Negativo }<0.25}{\text { Border-line } 0.25-0.35}$ \\
\hline $23 / 05 / 2003$ & & & Positivo $>0.35$ \\
\hline $23 / 05 / 2003$ & MEIA (S/CO) & Reattivo & $\mathbf{I . 5 9}$ & Positivo $>I .0$ \\
\hline $23 / 05 / 2003$ & ELFA & Positivo & $\mathbf{0 . 4 I}$ & Positivo $>0.35$ \\
\hline
\end{tabular}




\section{BIBLIOGRAFIA}

1. Aggarwal M, Rein J. Acute human immunodeficiency virus sindrome in an adolescent. Pediatrics 2003; 112 (4): 323-4.

2. Bourlet T, Pretis C, Pillet S, et al. Comparative evaluation of the VIDAS HIV DUO Ultra assay for combined detection of HIV-1 antigen and antibodies to HIV. J Vir Met 2005; 127 (2): 165-7.

3. Kobayashi S, Segawa S, Kawashima M, et al. A case of symptomatic primary HIV infection. J Dermatol 2005; 32 (2): 137-42.

4. Kwon JA, Yoon SY, Lee CK, et al. Performance evaluation of three automated human imuunodeficiency virus antigen-antibody combination immunoassays. J Virol Methods 2006; 133 (1): 20-6.

5. Ly TD, Laperche S, Courouce AM. Early detection of human immunodeficiency virus infection using thirdand fourth-generation screening assays. Eur J Clin Microbiol Infect Dis 2001; 20 (2): 104-10.

6. Rouzioux C, Chaix ML. Diagnosis and virologic monitoring of HIV infection. Rev Prat 1999; 49 (16): 1747-9.

Pasquale Minchella

U.O. Microbiologia e Virologia Azienda Sanitaria n. 6 Lamezia Terme Via A. Perugini, 1 - 88046 Lamezia Terme (CZ) Tel. 0968 208761; Fax 0968463113 e-mail: minchellap@libero.it 